\title{
ODD STARLIKE FUNCTIONS
}

\section{RAM SINGH AND SANGITA PURI}

\begin{abstract}
In the present paper, among other things, we prove that if $f(f(0)=0$, $\left.f^{\prime}(0)=1\right)$ is regular and odd starlike in $|z|<1$, then $\operatorname{Re} f(z) / s_{n}(z, f)>1 / 2$, $|z|<1$, where $s_{n}(z, f)$ denotes the $n$th partial sum of $f, n=1,2,3, \ldots$, thus generalising the known result: $\operatorname{Re} f(z) / z>1 / 2,|z|<1$. As an application, we show that each partial sum of an odd convex function is close-to-convex in $|z|<1$.
\end{abstract}

Introduction. Let $A$ denote the class of functions $f$ which are regular in the unit disc, $\mathscr{D}=\{z|| z \mid<1\}$, and are normalised by $f(0)=f^{\prime}(0)-1=0$. We denote by $S$ the subclass of $A$ consisting of univalent functions. For a given real number $\alpha$, $0 \leqslant \alpha<1$, let $S_{t}(\alpha)$ and $K(\alpha)$ represent the subclasses of $S$ consisting of starlike functions of order $\alpha$ and convex functions of order $\alpha$, respectively. $S_{t}(0)$ and $K(0)$ will be simply denoted by $S_{t}$ and $K$, and will, respectively, be referred to as the classes of starlike and convex functions. It is known that $K \subset S_{t}(1 / 2)$. Finally, let $C$ denote the subclass of $S$ made up of close-to-convex functions. A sufficient condition for $f \in A$ to be in $C$ is that $\operatorname{Re} f^{\prime}(z)>0$ in $\mathbf{D}$.

Marx [2] and Strohhäcker [7] proved independently that if $f \in K$, then $\operatorname{Re}(f(z) / z)>1 / 2, z \in \mathbf{D}$. Their result was generalised in two ways by Ruscheweyh and Sheil-Small [5] who proved that if $f \in S_{t}(1 / 2)$, then $\operatorname{Re}\left(f(z) / s_{n}(z, f)\right)>1 / 2$, $z \in \mathbf{D}$, where $s_{n}(z, f)$ denotes the $n$th partial sum of $f(z)$. It is also known that if $f \in A$ and $\operatorname{Re} f^{\prime}(z)>\alpha, \alpha<1$, then $\operatorname{Re}(f(z) / z)>\alpha, z \in \mathbf{D}$. For $f \in K$, Ruscheweyh [4] determined the radius of close-to-convexity of $s_{n}(z, f)$ depending upon $n$. Recently, Robertson [3] proved that if $f \in K(1 / 2)$, then all its partial sums $s_{n}(z, f)$ are close-to-convex in $\mathbf{D}$.

In the present short note we prove that if $f$ is an odd starlike function or $f \in A$ and satisfies the condition $\operatorname{Re} f^{\prime}(z)>1 / 2$ in $\mathbf{D}$, then $\operatorname{Re}\left(f(z) / s_{n}(z, f)\right)>1 / 2$, $z \in \mathbf{D}$. We also show that all partial sums of odd convex functions are close-to-convex in $\mathbf{D}$.

If $f(z)=\sum_{n=0}^{\infty} a_{n} z^{n}$ and $g(z)=\sum_{n=0}^{\infty} b_{n} z^{n}$ are regular in $|z|<r_{1}$ and $|z|<r_{2}$, respectively, then the function $f * g$, defined by the power series

$$
(f * g)(z)=\sum_{n=0}^{\infty} a_{n} b_{n} z^{n}
$$

Received by the editors April 19, 1984.

1980 Mathematics Subject Classification. Primary 30A26, 30A34.

Key words and phrases. Univalent, starlike, convex and close-to-convex functions, convolution/Hadamard product. 
is called the Hadamard product/convolution of $f$ and $g$, and is regular at least in the $\operatorname{disc}|z|<r_{1} r_{2}$.

We shall need the following results.

LEMMA 1. If $\phi$ and $g$ belong to $S_{t}(1 / 2)$, then for any function $F$, regular in $\mathbf{D}$, the function

$$
\frac{(\phi * F g)(z)}{(\phi * g)(z)} \quad(z \in \mathbf{D})
$$

takes values in the closed convex hull of $F(\mathbf{D})$.

Lemma 2. If $f \in A$ and satisfies the condition $\operatorname{Re} f^{\prime}(z)>1 / 2$ in $\mathbf{D}$, then for all points $z$ and $z_{0}$ in $\mathbf{D}$, we have

$$
\operatorname{Re} \frac{f(z)-f\left(z_{0}\right)}{z-z_{0}}>\frac{1}{2}
$$

Lemma 1 is due to Ruscheweyh and Sheil-Small [5] and Lemma 2 is an easy consequence of a result of Sakaguchi [6].

We now prove the following:

THEOREM 1. If $f(z)=z+a_{3} z^{3}+a_{5} z^{5}+\cdots$ is an odd starlike function, then for all $n \geqslant 1$, we have

$$
\operatorname{Re} \frac{f(z)}{s_{n}(z, f)}>\frac{1}{2} \quad(z \in \mathbf{D}) .
$$

Proof. Since $f(z)=z+a_{3} z^{3}+a_{5} z^{5}+\cdots$ is an odd starlike function, it is easily verified that the function $f_{1}$, defined by

$$
f_{1}(z)=\sqrt{z} f(\sqrt{z})=z+a_{3} z^{2}+a_{5} z^{3}+\cdots+a_{2 n-1} z^{n}+\cdots,
$$

is in $S_{t}(1 / 2)$.

Let $g(z)=z /(1-z)$. Then $g \in K \subset S_{t}(1 / \hat{2})$. Thus both $f$ and $g$ belong to $S_{t}(1 / 2)$. In view of Lemma 1 , we therefore conclude that the function

$$
\psi(z)=\frac{f_{1}(z) * g(z)\left(1-z^{n}\right)}{f_{1}(z) * g(z)}
$$

takes values in the closed convex hull of $F(\mathbf{D})$, where $F(z)=\left(1-z^{n}\right)$. In other words,

$$
|\psi(z)-1|<1 \quad(z \in \mathbf{D}),
$$

or,

$$
\left|\frac{z+a_{3} z^{2}+a_{5} z^{3}+\cdots+a_{2 n-1} z^{n}}{\sqrt{z} f(\sqrt{z})}-1\right|<1 \quad(z \in \mathbf{D})
$$

or,

$$
\left|\frac{s_{n}(\sqrt{z}, f)}{f(\sqrt{z})}-1\right|<1 \quad(z \in \mathbf{D})
$$


which implies, setting $\sqrt{z}=\xi$, that

$$
\operatorname{Re} \frac{f(\xi)}{s_{n}(\xi, f)}>\frac{1}{2} \quad(\xi \in \mathbf{D}) .
$$

This completes the proof of our theorem.

THEOREM 2. If $f(z)=z+a_{3} z^{3}+a_{5} z^{5}+\cdots$ is an odd convex function, then all partial sums $s_{n}(z, f)$ are close-to-convex in $\mathbf{D}$ with respect to $f$.

Proof. Since $f(z)$ is an odd convex function, $z f^{\prime}(z)$ is odd and starlike in D. Hence, in view of Theorem 1 , we conclude that for all $z \in \mathbf{D}$

$$
\operatorname{Re} \frac{z f^{\prime}(z)}{s_{n}\left(z, z f^{\prime}\right)}>\frac{1}{2}
$$

or, equivalently,

$$
\operatorname{Re} \frac{f^{\prime}(z)}{s_{n}^{\prime}(z, f)}>\frac{1}{2} \quad(z \in \mathbf{D}) .
$$

The last inequality implies that $\operatorname{Re}\left(s_{n}^{\prime}(z, f) / f^{\prime}(z)\right)>0$ in $\mathbf{D}$, and the desired conclusion follows.

TheOREM 3. If $f \in A$ and satisfies the condition $\operatorname{Re} f^{\prime}(z)>1 / 2$ in $\mathbf{D}$, then for all $n \geqslant 1$,

$$
\operatorname{Re} \frac{f(z)}{s_{n}(z, f)}>\frac{1}{2} \quad(z \in \mathbf{D}) .
$$

Proof. Let $f(z)=z+\sum_{n=2}^{\infty} a_{n} z^{n}$. Since $\operatorname{Re} f^{\prime}(z)>1 / 2$ in $\mathbf{D}$, in view of Lemma 2, we have for a given $z_{0} \in \mathbf{D}$,

$$
\operatorname{Re} \frac{f(z)-f\left(z_{0}\right)}{z-z_{0}}>\frac{1}{2} \quad(z \in \mathbf{D}),
$$

or,

$$
\operatorname{Re}\left[\frac{f\left(z_{0}\right)}{z_{0}}+\sum_{n=1}^{\infty} \frac{f\left(z_{0}\right)-s_{n}\left(z_{0}, f\right)}{z_{0}^{n+1}} z^{n}\right]>\frac{1}{2} \quad(z \in \mathbf{D}),
$$

from which it follows that

$$
\left|\frac{f\left(z_{0}\right)-s_{n}\left(z_{0}, f\right)}{z_{0}^{n+1}}\right| \leqslant \operatorname{Re} \frac{f\left(z_{0}\right)}{z_{0}} \leqslant\left|\frac{f\left(z_{0}\right)}{z_{0}}\right| \quad[1, \mathrm{p} .41]
$$

Therefore,

$$
\left|\frac{s_{n}\left(z_{0}, f\right)}{f\left(z_{0}\right)}-1\right| \leqslant\left|z_{0}\right|^{n}<1,
$$

or equivalently,

$$
\operatorname{Re} \frac{f\left(z_{0}\right)}{s_{n}\left(z_{0}, f\right)}>\frac{1}{2} .
$$

This completes the proof of our theorem. 


\section{REFERENCES}

1. P. L. Duren, Univalent functions, Springer-Verlag, New York, 1983.

2. A. Marx, Untersuchungen über schlichte Abbildungen, Math. Ann. 107 (1932-33), 40-67.

3. M. S. Robertson, Univalent functions starlike with respect to a boundary point, J. Math. Anal. Appl. 81 (1981), 327-345.

4. St. Ruscheweyh, On the radius of univalence of the partial sums of convex functions, Bull. London Math. Soc. 4 (1972), 367-369.

5. St. Ruscheweyh and T. Sheil-Small, Hadamard products of schlicht functions and the Pólya-Schoenberg conjecture, Comment. Math. Helv. 48 (1973), 119-135.

6. K. Sakaguchi, On a certain univalent mapping, J. Math. Soc. Japan 11 (1959), 72-75.

7. E. Strohhäcker, Beiträge zur Theorie der schlichten Funktionen, Math. Z. 37 (1933), 356-380.

Department of Mathematics, Punjabi University, Patiala - 147002, India 